\title{
Wie verändert Technik das Alter(n) und die Gerontologie?
}

\author{
Laura Schmidt \& Hans-Werner Wahl
}

Das noch junge Feld der gero-technologischen Forschung ist durch die Überlagerung der gesellschaftlichen Trends des demographischen Wandels und der zunehmenden Technisierung begründet und geprägt. Wir stellen relevante theoretische Zugänge und einige ausgewählte empirische Befunde dar und diskutieren den Beitrag und die Bedeutung von Technik für das Altern und die Gerontologie.

\section{Comment la technologie peut-elle modifier la vieil- lesse et la gérontologie ? \\ Le domaine encore récent de la recherche en géron- technologie est fondé sur la superposition des ten- dances sociales de l'évolution démographique et de la technicité croissante qui le caractérisent. Nous allons présenter des accès théoriques pertinents et quelques découvertes empiriques choisies et dis- cuter de la contribution et de la signification de la technologie en matière de vieillissement et de gérontologie.}

DOI 10.1024/2297-5160/a000004

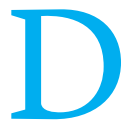
ie Überlagerung der allseits bekannten demographischen Trends mit jenen der exponentiell fortschreitenden Übergänge unserer Gesellschaften in zunehmend digitalisierte und virtualisierte «Welten» wird bislang noch $\mathrm{zu}$ wenig explizit in den gerontologischen Forschungs- und Anwendungsblick genommen.

\section{Technik und Alter(n) noch primär als «Frage»-Thematik ohne profunde Antworten}

Was werden am Ende die Ergebnisse dieser Überlagerung sein? Werden Altern und ältere Menschen sowie ihre Sozialpartner, auch Pflegepersonen, durch eine breit aufgestellte Nutzung von Technologie in zwei bis drei Jahrzehnten besser dastehen - oder eher nicht? Ist die bisweilen zu findende Euphorie mancher Entwickler und auch $A A L$ begeisterter ${ }^{1}$ Forschender tatsächlich berechtigt? Oder

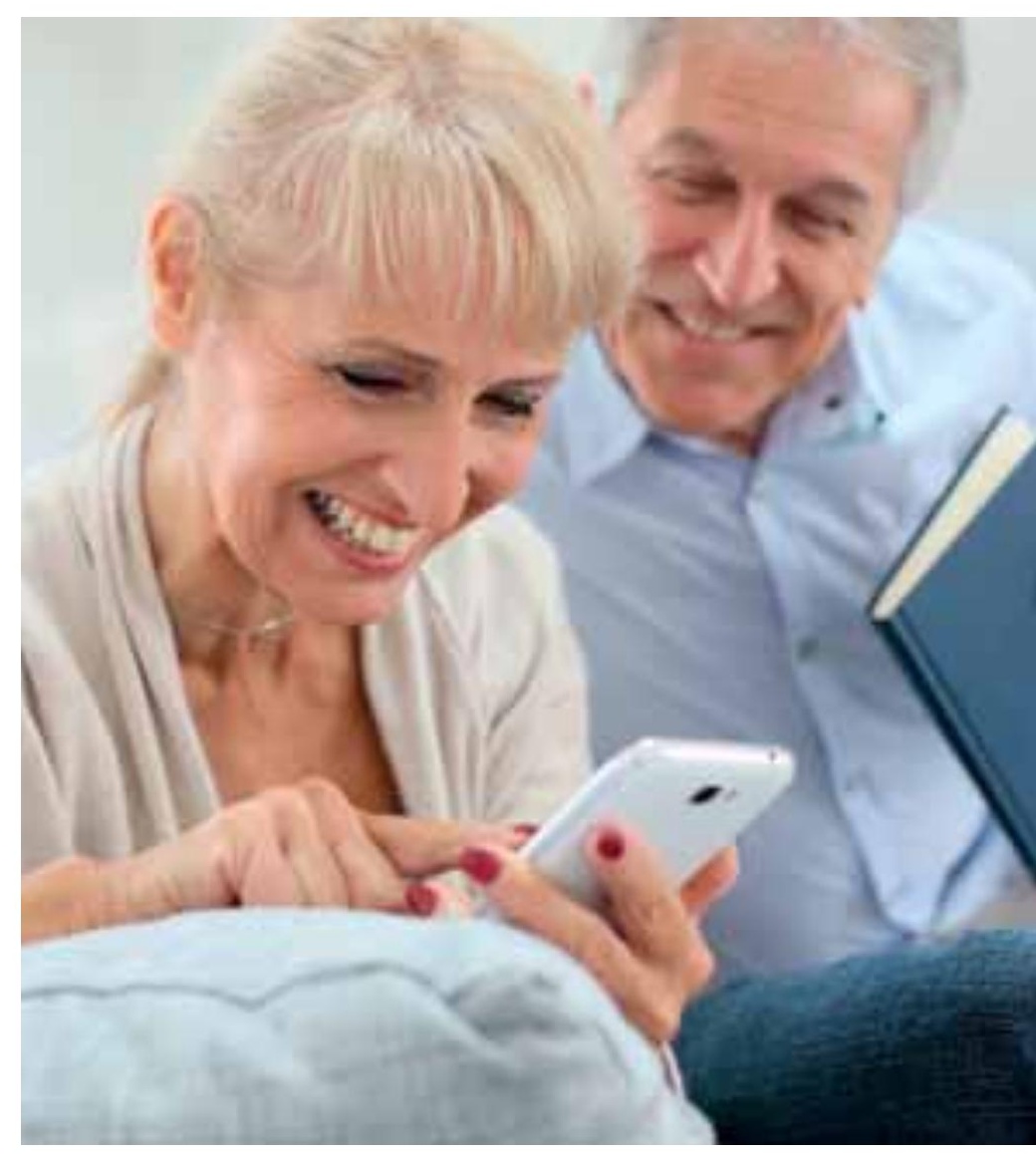

ganz konkret: Ist die Nutzung eines Smartphones oder eines Robotertieres im fortgeschrittenen Alter in welcher Weise auch immer per se schon ein Fortschritt? Oder eher ein Rückschritt? Wird das Thema Alter und Technik (wir sprechen im Folgenden von Gero-Technologie) auch die Gerontologie als Wissenschaft befördern?

Klar ist heute zumindest, dass sich die Forschungsthematik der Gero-Technologie (auch) in der Gerontologie zunehmend etabliert hat und wissenschaftliche und anwendungsbezogene «Ausstrahlungen» sucht. Allerdings werden aus unserer Sicht profunde Antworten auf die eben aufgeworfenen Fragen noch auf sich warten lassen; dafür ist das Feld zu jung und wohl auch zu wenig konzeptuell und empirisch entwickelt. Dennoch zeichnen sich aus unserer Sicht erste Umrisse für solche Antworten ab.

$A A L=$ Ambient Assisted Living 


\section{Wichtige theoretische Zugänge und empirische Befunde}

Wir möchten zunächst argumentieren, dass es höchst unklug auch im Bereich Gero-Technologie wäre, Unmengen von Daten und Befunden zu generieren, aber diese am Ende nicht wirklich zu verstehen. Wir sehen eine solche Tendenz durchaus und gehen aus diesem Grund zunächst auf Theorien mit Bedeutung für Gero-Technologie ein.

\section{Theoretische Zugänge}

Der Einsatz von Technik bietet für ein erfolgreiches Altern vielfältige Potentiale. Das in der Psychologischen Alternsforschung etablierte Modell der Selektiven Optimierung mit Kompensation (SOK) beruht auf der altersbedingten Verringerung der Ressourcen des Organismus (Baltes, 1997) und kann gut auf die Techniknutzung übertragen werden: Diese kann (1) die Selektion unterstützen, z. B. hilft bei einer Mobilitätsbeeinträchtigung der Internetauftritt des Nahverkehrs, die barrierefreien Haltestellen zu finden, (2) im Sinne der Kompensation dem Ausgleich nachlassender körperlicher und kognitiver Fähigkeiten dienen oder (3) diese Funktionen optimieren und entwicklungsförderliches Potenzial bieten, z. B. hinsichtlich der Selbstständigkeit oder Mobilität (Claßen et al., 2014).

Lindenberger und Kollegen entwickelten auf Basis der SOK-Strategien drei psychologische Prinzipien zur Evaluation von Technik (Lindenberger et al., 2008). Net Resource Release bedeutet im Sinne einer "positiven Ressourcenbilanz», dass die Techniknutzung nur dann adaptiv ist, wenn die Bedienungskosten niedriger sind als der Nutzen bezüglich der freigesetzten kognitiven Ressourcen, wobei sowohl auf objektive als auch subjektive Einschätzungen zurückgegriffen werde sollte. Das zweite Prinzip der Personenspezifität betont die Relevanz der Anpassung der Technik an individuelle Gewohnheiten und Fähigkeiten der älteren Nutzer, wobei sowohl Heterogenität als auch intraindividuelle Variabilität beachtet werden sollen - im Idealfall durch «lernende» Systeme. Das dritte Prinzip des proximalen vs. distalen Beurteilungsrahmens beschreibt die Abhängigkeit der Potentiale und Risiken von der betrachteten Zeitspanne. Zum Beispiel kann durch Techniknutzung Belastung oder Anstrengung reduziert werden und diese freigewordenen Ressourcen können anderweitig genutzt werden. Auf der anderen Seite könnten bestimmte Fähigkeiten durch «disuse» (Nicht-Nutzung) abnehmen; man denke z.B. an eine ständige Nutzung von Navigationssystemen, der möglicherweise ein Rückgang der Orientierungsfähigkeit folgt.

In Lawtons Rahmenmodell zur Person-Umwelt-Passung (Lawton, 1998) wird angenommen, dass geringe Ressourcen auf Seiten der Person - man denke z. B. an eine (leichte) kognitive Beeinträchtigung, sensorische Einbussen oder geringe Technikerfahrung - die Wahrscheinlichkeit erhöhen, dass Umweltfaktoren (hier: technische Systeme) das Verhalten kontrollieren, dass jedoch grössere verfügbare Ressourcen ein proaktives Nutzen der Umweltgegebenheiten bzw. der Technik ermöglichen.
Schliesslich existieren auf dem Gebiet der Technikakzeptanzforschung zum Teil eng verwandte Theorien wie das weitverbreitete Technology Acceptance Model (TAMTAM3; Davis, 1989), das Technology Readiness and Acceptance Model (TRAM; Lin, Shih, \& Sher, 2007) oder die Unified Theory of Acceptance and Use of Technology (UTAUT; Venkatesh et al., 2003), welche Nutzungsintentionen auf Basis von Einschätzungen zur Nützlichkeit und Leichtigkeit der Nutzung sowie generellen Überzeugungen und Einstellungen bezüglich Technik erklären.

\section{Empirische Befunde}

Die Forschungslandschaft zu Gero-Technologie lässt sich zum einen auf Outcome-Ebene in die Bereiche der Technikausstattung, der Akzeptanzforschung und der konkreten Technikhandhabung bzw. Performanzforschung gliedern (Schmidt, 2015; Schmidt et al., 2014). Zum anderen sollten relevante Nutzergruppen unterschieden werden, z.B. unbeeinträchtigte Ältere, Personen mit leichter kognitiver Beeinträchtigung (Mild Cognitive Impairment, MCI) und Personen mit Demenz bzw. deren pflegende Angehörige oder professionelle Personen im Heimkontext.

Einzelne Komponenten des TAM wurden zuletzt verstärkt auch in der Gerontologie und der psychologischen Alternsforschung untersucht, sowohl in Deutschland (Claßen, 2012; Mollenkopf \& Kaspar, 2005) als auch auf europäischer Ebene und international (Czaja et al., 2006; Mitzner et al., 2010; Tacken et al., 2005). Insgesamt kann die Rolle von Einstellungs- und Bewertungsdimensionen auf die Technikakzeptanz und die Techniknutzung als bestätigt gewertet werden; teilweise wurde das TAM durch Einbezug psychologischer Konstrukte wie Persönlichkeit und SOK-Strategien ergänzt (Claßen, 2012) oder um kontextuelle Faktoren wie den Mediendiskurs, soziale Einflüsse und die Preisgestaltung erweitert (Baaren, van de Wijngaert \& Huizer, 2011). In Forschungsarbeiten zur Computerakzeptanz und Internetnutzung werden zudem häufig spezifische computer- oder internetbezogene Selbstwirksamkeitserwartungen erfragt (Chung et al., 2010; Wild et al., 2012) für die dann überwiegend negative Zusammenhänge mit dem Lebensalter und positive Beziehungen zur Nutzungsintention gefunden werden.

Im Vergleich zur Akzeptanzforschung sind die Forschungsbestrebungen zur konkreten Technikhandhabung selten theoriebasiert; häufig beruhen die Studien auf kleinen experimentellen Ansätzen, in denen junge, meist studentische Probanden mit älteren bezüglich bestimmter Performanzkriterien (z.B. Fehler, Bearbeitungszeit) im Umgang mit v.a. Computer- und Internetanwendungen verglichen werden. Hochwertige Studien hinsichtlich der Rolle kognitiver Faktoren finden sich im Umfeld des Center for Research and Education on Aging and Technology Enhancement (CREATE). Beispielsweise konnten für Aufgaben zur Dateneingabe, Textverarbeitung, Informationssuche oder Website-Navigation bedeutsame Erklärungsbeiträge bestimmter Facetten wie Arbeitsgedächtnis, schlussfolgerndes Denken, Exekutivfunktionen, Verarbei- 
tungsgeschwindigkeit und räumliches Vorstellungsvermögen belegt werden (z.B. Czaja et al., 2013; Ownby et al., 2008; Taha et al., 2013).

Neben der Kognition wird die Bedeutung von Überzeugungen erforscht: So belegen Arning und Ziefle (2007) einen positiven Zusammenhang von Kontrollüberzeugung und der Leistung bei Aufgaben mit einem digitalen Tagebuch (PDA); bei Schmidt (2015) trug die generalisierte Selbstwirksamkeitserwartung über die Kognition hinaus zur Erklärung der Performanz mit Alltagstechnik (Blutdruckmessgerät, Mobiltelefon und E-Book Reader) bei.

Während die oben beschriebene Forschung zum grössten Teil höhergebildete und technikaffine Ältere im sogenannten „Dritten Alter» einschliesst, besteht noch Forschungsbedarf hinsichtlich der Gruppe von Personen mit MCI, die ca. $16 \%$ der Bevölkerung über 65 Jahren ausmachen (Kaduszkiewicz et al., 2014). Es gibt Hinweise, dass sich Personen mit MCI hinsichtlich der subjektiv empfundenen Schwierigkeit bei der Technikhandhabung zwischen kognitiv gesunden Älteren und Personen mit leichter Demenz einordnen lassen (z.B. Nygård et al., 2012). Neben Gruppendifferenzen von mittlerer bis grosser Effektstärke im Vergleich zu unbeeinträchtigten Älteren konnte Schmidt (2015) insbesondere für Probanden mit MCI eine hohe Relevanz fluider und gedächtnisbezogener Komponenten für den Umgang mit Alltagstechnik belegen. Darüber hinaus spielte das persönlichkeitsnahe Einstellungsmass der erlebten Obsoleszenz , das Entfremdungs- und Orientierungsprobleme umfasst, die sich für ältere Menschen aus der Dynamik des gesellschaftlichen Wandels ergeben können (Brandtstädter \& Wentura, 1994), eine bedeutsame Rolle für den erfolgreichen Technikumgang.

\section{Resümee 1: \\ Wie verändert Technik das Alter(n)?}

Man kann bei der Antwortsuche auf diese Frage mindestens zwei Ebenen unterscheiden: Zum ersten spricht vieles dafür, dass Technik das alltägliche Leben älterer Menschen, letztlich ihre Lebensqualität, vielschichtig beeinflusst. Zum zweiten stellt die zunehmende Nutzung von Technologien selbst einen Faktor dar, der das Alter verändert. In Bezug auf den ersten Aspekt nennt Mollenkopf (2000) fünf Kategorien von besonders für ältere Menschen geeigneter Technik, nämlich Technik (1) zur Prävention oder Verzögerung alterskorrelierten Nachlassens von Stärke, Flexibilität und Ausdauer im physiologischen, kognitiven oder sozialen Bereich, (2) zur Verbesserung und Stärkung spezifischer Bereiche oder neuer Rollen, (3) zur Kompensation nachlassender Fähigkeiten im Alter, (4) zur Unterstützung Pflegender und (5) zur Verbesserung der gerontologischen Forschung. Schulz und Kollegen etablierten den Begriff der «Quality of Life Technologies», der (nur) solche Systeme umfasst, die die Lebensqualität der (älteren) Nutzer unmittelbar positiv beeinflussen (Schulz et al., 2015). Dieses breite Begriffsverständnis schliesst nicht nur kompensatorische Systeme (wie Assistenztechnik) ein, sondern umfasst ebenso Technik mit präventivem Charakter, die der Unterhaltung, Stimulation, sozialen Eingebundenheit, Informationssuche und Aktivität dienen kann. Forschung muss letztlich zeigen, ob und in welcher Weise jeweils fokussierte Technologien in der Lage sind, die genannten Bereiche tatsächlich zu unterstützen und damit, in der Sprache der Geropsychologie, auch Entwicklungsprozesse im höheren Lebensalter $\mathrm{zu}$ fördern. Wenn beispielsweise ein Roboter bei einem hochaltrigen, multimorbiden und im Gehen stark beeinträchtigten Menschen Anforderungen im häuslichen Alltag vielfältig, effizient und bedürfnisgerecht unterstützt, dann könnte es sein, dass dadurch Ressourcen freigesetzt werden, die diesem Menschen nun erlauben, Bildungsziele («Englisch lernen») oder soziale Kontakte («WhatsApp») via Internet zu verwirklichen.

Hinsichtlich des zweiten Aspekts haben Mollenkopf et al. (1999) schon früh argumentiert, dass umfassende Techniknutzung im Alter möglicherweise deutliche Auswirkungen im Hinblick auf das Erleben von Autonomie, Selbstwirksamkeit und Gesundheit haben könnte, also auf Bereiche, die alle negativ mit Alter korreliert sind. Geeignete Technologien könnten dazu führen, dass typische Verluste der Selbständigkeit deutlich abgemildert werden, verbunden mit einem stärker «kontextuellen» Verständnis von Technik: So lange die Technik mir hilft, meine Handlungsziele zu erreichen, bin ich selbständig, auch wenn ich dies ohne Technik nicht mehr könnte. In ähnlicher Weise könnten sich auch Auswirkungen auf Gesundheitsbewertungen ergeben bzw. sogar ein neues Verständnis von Gesundheit entstehen, dass die unterstützende und fördernde Rolle von Technik miteinbezieht. Daneben könnten neue Technikkompetenzen eben das Kompetenzund Selbstwirksamkeitserleben im Alter insgesamt stärken. Wahrscheinlich werden die Älteren von morgen zudem in grossem Stil auch "Tracking»- und auf die unmittelbare Erfassung biologischer Funktionen abzielende Systeme nutzen, darauf aufbauend ihre Gesundheit regulieren und sich etwa Handlungsvorschläge im Hinblick auf die Art und Intensität von Trainingsprogrammen oder die Konsultation von Professionellen (Ärzte, Psychologen, Ergotherapeuten etc.) einholen.

\section{Resümee 2: \\ Wie verändert Technik die Gerontologie?}

Wenig ist bislang danach gefragt worden, ob und wie die zunehmende Etablierung der Gero-Technologie die Alternsforschung selbst verändern wird. Erstens sind die Entwicklungen bislang noch kaum im Theoriebestand der Gerontologie verankert (Schulz et al., 2015) mit wenigen Ausnahmen: Wie wir weiter oben gesehen haben, ist das SOK-Modell vor allem von Lindenberger et al. (2008) auch in die technische Sphäre ausgedehnt worden, was zu einer Reihe von neuen Forschungsfragen in Bezug auf dieses Modell von Entwicklungsregulation führt. Auch könnte es interessante Verschränkungen mit der sozio-emotionalen Selektivitätstheorie (Carstensen, 2006) geben, etwa in- 
dem heute neue Kommunikationsformen via Skype oder WhatsApp die von Älteren präferierten nahen und intimen Sozialkontakte und damit die sozio-emotionale Selektivität direkt unterstützen können. Wahl und Kollegen (2012) argumentieren sogar, dass wir in Zukunft gerade im Bereich von Belonging Prozessen, die vor allem für Ältere grosse Bedeutung besitzen (Was ist mir wichtig? Wo gehöre ich hin?), auch immer mehr Technikaspekte sehen werden, etwa in Gestalt von sog. emotionaler Robotik, dem «stimmungsaufhellenden» Robotertier etwa, oder der «Liebe» zur smart ausgestatteten Wohnung, ohne die man schon längst im Pflegeheim wohnen würde.

Eine zweite Implikation besteht darin, dass Gero-Technologie der Gerontologie eine weitere, aktuell sehr bedeutsame Tür öffnet, sich mit grundlegenden Veränderungen unserer Gesellschaft zu vernetzen bzw. diese mitzugestalten. Das scheint uns auch für die Weiterentwicklung der Gerontologie sowie deren gesellschaftliche Rolle sehr bedeutend zu sein.

Forschungsseitig sollte dies drittens bedeuten, dass die Nutzung und das Erleben von Technologien zukünftig in jedes Datenprotokoll von grossen Studien gehören. Dies wird es einmal ermöglichen, Techniknutzung mit anderen Konstrukten, die für die Alternsforschung zentral sind (z.B. Einsamkeit, soziale Unterstützung, kognitive Leistung, Depressivität, Mobilität), in Beziehung zu setzen, und dies natürlich nach Möglichkeit längsschnittlich. Insofern appellieren wir an die Projektleiter laufender Längsschnittstudien in der Alternsforschung, aber auch darüber hinaus (z. B. Sozio-ökonomisches Panel) einige Grundindikatoren zur (Gero-)Technologienutzung einzubeziehen (z. B. Nutzungsprofil, Nutzungshäufigkeit, erlebter Nutzen, eventuell auch Gründe für Nichtnutzung und erlebte Schwierigkeiten).

Viertens werden die neuen Technologien auch in der Gerontologie die Daten-Assessment-Strategien revolutionieren (s.o.: Beispiel zu Tracking). Mit ambulatorischen Messungen auf vielen Ebenen (Selbstbericht, Bewegung, Bio-Indikatoren, Ortung im Raum, automatisierte Sprachanalyse etc.) werden Gerontologie-Forscher schon sehr bald vor riesigen Datenquantitäten stehen, die neue Liaisons mit mathematischer Kompetenz unabdingbar machen (Brose \& Ebner-Priemer, 2015; Gerstorf, Hoppmann \& Ram, 2014). Es versteht sich fast von selbst, dass mit diesen Entwicklungen gewichtige ethische Fragen (auch) die Alternsforschung einholen werden, etwa Fragen nach unkontrollierter Überwachung, nach Datenschutz generell, vor allem aber bei sehr vulnerablen alten Menschen (Wahl et al., 2010).

\section{Technik und Alter: Ein unter- und überschätztes Thema}

Insgesamt kommen wir zum Schluss, dass das Thema der Gero-Technologie grosse Bedeutung für die Alternsforschung und ihre gesellschaftliche Relevanz, aber auch für zukünftiges Leben im Alter besitzt. Dennoch sind dies bis zu einem gewissen Grade Vermutungen, denn die eigentliche Forschungslandschaft zu Gero-Technologie beginnt zwar Gestalt anzunehmen, ist aber von einem etablierten, robusten, stark interdisziplinär vernetzten und heuristisch fruchtbaren Forschungsprogramm noch weit entfernt. Äusserungen, diese Forschungen wären doch derzeit noch «heisse Luft», basierend auf kleinen Fallzahlen und noch zu wenig entwickelten Theorien, halten wir für eine klare Unterschätzung dessen, was sich im Bereich Gero-Technologie bereits an wertvollen Impulsen entwickelt hat. Auf der anderen Seite darf aber das zarte «Forschungs-Pflänzchen», das sich entwickelt hat, nicht mit einem «Forschungsbaum» verwechselt werden. Hier gibt es bisweilen auch gravierende Überschätzungen der Bedeutung des Gebiets.

\section{Auswahl an Literatur:}

Schmidt, L. I. (2015). Technikhandhabung im höheren Alter aus psychologischer Perspektive: Eine quasiexperimentelle Studie zur Rolle von kognitiver Leistungsfähigkeit, Technikeinstellung und Technikerfahrung. Dissertation, Universität Heidelberg.

Wahl, H.-W., Iwarsson, S., \& Oswald, F. (2012). Aging well and the environment: Toward an integrative model and research agenda for the future. The Gerontologist, 52, 306-316. doi: 10.1093/geront/gnr154.

(Die vollständige Literatur ist bei den Autoren zu erhalten)

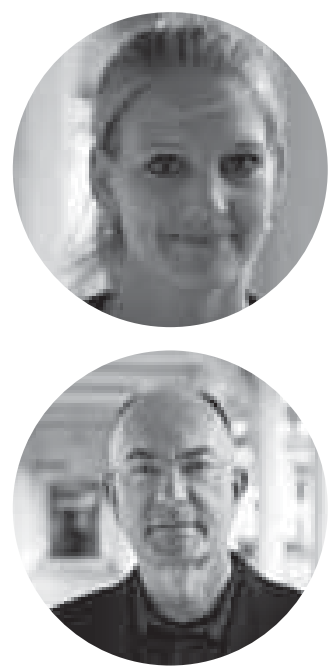

Dr. Laura Schmidt ist Psychologin und arbeitet als wissenschaftliche Mitarbeiterin im Team von HansWerner Wahl.

Prof. Dr. Hans-Werner Wahl leitet die Abteilung für Psychologische Alternsforschung am Psychologischen Institut der Universität Heidelberg, Deutschland.

h.w.wahl@psychologie. uni-heidelberg.de 